\title{
Estimación de parámetros de curvas de crecimiento de ganado Nellore criado en confinamiento
}

\section{Estimating parameters of growth curves in Nellore cattle raised in confinement}

\author{
Sandra Posada O, ${ }^{1 *}$ Ph.D, Ricardo Rosero N, ${ }^{1}$ Ph.D, Norberto Rodríguez, ${ }^{2}$ Ph.D, \\ Ana Costa $C_{1}^{2}$ Ph.D.
}

\begin{abstract}
${ }^{1}$ Universidad de Antioquia, Facultad de Ciencias Agrarias, Grupo de Investigación en Ciencias Agrarias, GRICA, Medellín, Colombia. ${ }^{2}$ Universidade Federal de Minas Gerais, Escola de Veterinária, Belo Horizonte, Brasil. *Correspondencia:slposada@gmail.com.
\end{abstract}

Recibido: Septiembre de 2010; Aceptado: Julio de 2011.

\section{RESUMEN}

Objetivo. Comparar los modelos de regresión no lineal Brody, Gompertz, Logístico y von Bertalanffy, e identificar cual de ellos describe mejor el crecimiento de machos enteros Nellore criados en régimen de confinamiento, recibiendo suplementación alimenticia y sometidos a manejo experimental. Materiales y métodos. La estimativa de los parámetros de cada modelo se realizó a través del procedimiento para modelos no lineales PROC NLIN de SAS, a partir de datos de peso colectados desde los 352 hasta los 833 días de edad. Resultados. El cuadrado medio del error, el coeficiente de determinación y la dócima de Durbin-Watson permitieron concluir que todas las funciones presentaron comportamiento similar en su capacidad de ajuste; no obstante, el análisis de normalidad de residuos demostró que el modelo logístico no cumplió con este supuesto $(p<0.05)$, limitando su habilidad predictiva bajo las actuales condiciones experimentales. Conclusiones. La condición de normalidad en los residuos de los modelos de Brody, Gompertz y von Bertalanffy $(p>0.05)$ permitió asegurar que la inferencia sobre los parámetros fue adecuada, con independencia de los cambios de peso resultantes de la aplicación de diversos planos alimenticios. Por la interpretación biológica de los parámetros, el modelo Brody fue el que presentó la mejor descripción del crecimiento de machos Nellore. Los mayores pesos maduros y las menores tasas de madurez obtenidas en este trabajo, en relación con los reportes de la literatura, indica que los animales alcanzarían peso asintótico en edad más avanzada en razón de su desarrollo más lento, consecuencia del manejo experimental al que fueron sometidos.

Palabras clave: Biomodelación, bondad de ajuste, masa corporal, regresión no lineal (Fuente: $C A B)$. 


\section{ABSTRACT}

Objective. To compare the Brody, Gompertz, logistic and von Bertalanffy nonlinear regression models in their ability to describe the growth in Nellore cattle raised in confinement, receiving a feed supplement and subjected to experimental manipulation. Materials and methods. The parameters for each model were estimated using the procedure for nonlinear models PROC NLIN of SAS, based on weight data collected from 352 to 833 days of age. Results. Mean square error, coefficient of determination and Durbin-Watson statistic allowed to concluding that all functions had similar ability to adjust the weightage data observed; however, the normality test for the residuals showed that the logistic model was the one that did not meet this assumption $(p<0.05)$, limiting its predictive ability under current experimental conditions. Conclusions. The normality condition in the residuals of Brody, Gompertz and von Bertalanffy models ( $p>0.05)$ ensured that the inference about the parameters was adequate, regardless of weight changes resulting from the implementation of various feeding levels. For the biological interpretation of the parameters, the Brody model was the one that best described the recorded growth in Nellore males. The highest mature weight and the lowest mature index obtained indicate that animals reached asymptotic weight at older age because of their slower growth, as consequence of their manipulation during the experiment.

Key words: Biomodeling, goodness of fit, body mass, nonlinear regression (Source: $C A B$ ).

\section{INTRODUCCIÓN}

El estudio del crecimiento, como función biológica sujeta a la interacción genotipoambiente, resulta importante al estar directamente relacionado con la cantidad y la calidad de la carne producida $(1,2)$. Además, permite identificar los animales que presentan mayor peso en menor edad (3), que se traduce en mayor precocidad (4) y máximo beneficio económico para la empresa ganadera (5).

El aumento de masa corporal de un animal ocurre en una secuencia temporal: prenatal, posnatal hasta el destete, destete hasta la pubertad $y$, pubertad hasta la madurez, donde cada fase presenta diferente velocidad o tasa de crecimiento. El análisis de esas tasas es de interés para investigadores y productores porque indica cuales son las necesidades nutricionales y ambientales de los animales en cada fase, permite evaluar la eficiencia del crecimiento animal y provee información para elaborar programas de mejoramiento genético (1).

Datos provenientes de medidas longitudinales del peso corporal se distribuyen de forma semejante a curvas exponenciales y pueden ser analizados por modelos matemáticos no lineales, los cuales han sido desarrollados empíricamente para relacionar datos peso-edad y se han mostrado adecuados para describir curvas de crecimiento $(2,6)$. Algunos requisitos para que una función de crecimiento sea aceptada como descriptiva de la relación peso-edad son interpretación biológica de los parámetros, ajuste con pequeños desvíos y facilidad de convergencia (6).

Generalmente, las funciones de crecimiento presentan tres parámetros interpretables biológicamente y otro que se identifica como una constante matemática. El parámetro A, definido como peso asintótico o peso adulto, representa la estimativa de peso a la madurez, independiente de fluctuaciones de peso debidas a efectos genéticos y ambientales (6), cuando el tiempo tiende a infinito (3). El parámetro $\mathrm{K}$, que se conoce como índice de madurez, estimativa de precocidad de madurez o tasa de madurez posnatal, determina la eficiencia del crecimiento de un animal. Es la razón entre la tasa de crecimiento máxima y el peso adulto del animal. Cuanto mayor sea 
el valor de este parámetro más precoz es el animal, en tanto que valores más bajos indican madurez tardía, por tanto representa un indicador de la velocidad con que el animal se aproxima al peso adulto $(1,6)$. El inverso de $\mathrm{K}\left(\mathrm{K}^{-1}\right)$ corresponde al tiempo necesario para alcanzar la madurez (7). El parámetro $M$ es denominado parámetro de inflexión y se refiere al punto en que el animal pasa de una fase de crecimiento acelerado a una fase de crecimiento inhibitorio, o lo que es lo mismo, el punto a partir del cual el animal pasa a crecer con menor eficiencia. Excepto en la función de Richards, este parámetro asume valores fijos, haciendo que las funciones presenten formas definidas. El parámetro $b$ es denominado parámetro de integración o intercepción con el eje $Y$, no posee significado biológico y es utilizado apenas para adecuar el valor inicial del peso vivo, haciendo con que la curva pase por el origen cuando $y \neq 0$ y/o $t \neq 0(6,7)$.

La evaluación del crecimiento permite analizar y gerenciar la rentabilidad de un sistema de producción de carne y la mayoría de los reportes obtenidos con ganado cebuíno en condiciones tropicales han sido generados bajo sistemas en pastoreo (1), por lo tanto el presente trabajo pretende comparar los modelos de regresión no lineal Brody, Gompertz, Logístico y von Bertalanffy e identificar cual describe mejor el crecimiento de machos enteros Nellore criados en régimen de confinamiento, recibiendo suplementación alimenticia y sometidos a manipulación experimental.

\section{MATERIALES Y MÉTODOS}

Tipo de estudio. Los datos utilizados en este trabajo provienen de un experimento de estimación de exigencias energéticas realizado durante cuatro períodos experimentales diferentes, correspondientes a intervalos de peso de cerca de $100 \mathrm{Kg}$ (desde los 200 hasta los $472 \mathrm{Kg}$ ). La capacidad de ajuste de las funciones se evaluó con datos de peso obtenidos desde los 352 hasta los 833 días de edad (11.6 a 27.4 meses).
Sitio de estudio. El experimento fue conducido en el Laboratorio de Metabolismo y Calorimetría Animal de la Escuela de Veterinaria de la Universidad Federal de Minas Gerais, Belo Horizonte (Brasil), entre junio de 2008 y enero de 2010. La ciudad de Belo Horizonte está situada a $900 \mathrm{msnm}$, posee una temperatura media de $23^{\circ} \mathrm{C}$, variando entre 9 y $35^{\circ} \mathrm{C}$, humedad relativa del $65 \%$ y precipitación promedio anual de $1600 \mathrm{~mm}$, con mayor presencia de lluvias entre octubre y marzo, tratándose de un clima tropical de altitud según la clasificación de Köppen (8). Geográficamente se encuentra a 190 '55' $15^{\prime \prime}$ de latitud Sur y $43^{\circ} 56^{\prime} 16^{\prime \prime}$ de longitud occidente, con respecto al meridiano de Greenwich.

Animales e instalaciones experimentales. Cinco cebuínos enteros de raza $\mathrm{Ne}-$ llore, con peso vivo medio inicial de $180 \mathrm{~kg}$ de peso, fueron mantenidos en régimen de confinamiento en galpón cubierto, en vallas individuales $(2 \times 1.5 \mathrm{~m})$, con piso de concreto revestido de tapete, comederos $y$ bebederos individuales.

Alimentación. La dieta estuvo constituida por heno de Tifton 85 (Cynodon spp.), suplemento concentrado a base de maíz y torta de soya y sal mineralizada comercial. La proporción forraje-concentrado varió en función de la edad de los animales, buscando una composición nutricional que permitiera obtener ganancias medias próximas a $700 \mathrm{~g} / \mathrm{animal} /$ día, de acuerdo con las recomendaciones de Valadares et al (9) (Tabla 1).

La ración fue suministrada ad libitum dos veces al día, a las 8 y 17 horas, en la forma de ración completa. La cantidad de alimento fue diariamente ajustada permitiendo un rechazo de entre 5 y $10 \%$ de lo ofrecido. $L a$ ración fue la misma para todos los animales, variando sólo las cantidades garantizadas de acuerdo con el manejo previamente expuesto. El consumo de materia seca (CMS) registrado en los períodos $1,2,3$ y 4 fue $2.52,1.98,1.88$ y $1.68 \%$ del peso vivo, respectivamente. Expresado en g/ Kg PV0.75/ día, los valores para los correspondientes períodos experimentales fueron 95.76, $83.56,82.99$ y $77.50 \mathrm{~g}$. 
Tabla 1. Contenido de materias primas (porcentaje) y composición química (porcentaje de la materia seca) de la ración ofrecida.

\begin{tabular}{lcccc}
\hline \multirow{2}{*}{ Ingredientes $^{\mathbf{1}}$} & \multicolumn{5}{c}{ Período } \\
& $\mathbf{1}$ & $\mathbf{2}$ & $\mathbf{3}$ & $\mathbf{4}$ \\
\hline Heno (Cynodon spp.) & 60 & 60 & 70 & 80 \\
Maíz & 20 & 25 & 18 & 10 \\
Torta de soya & 20 & 15 & 12 & 10 \\
Composición química $^{2}$ & & & & \\
MS & 89.73 & 88.87 & 89.62 & 90.11 \\
PB & 14.49 & 15.13 & 11.82 & 14.45 \\
EE & 1.58 & 2.40 & 2.17 & 2.06 \\
MI & 5.42 & 5.80 & 6.47 & 6.37 \\
FDNp & 55.51 & 48.40 & 56.72 & 65.08 \\
FDA & 27.31 & 22.15 & 27.68 & 32.09 \\
Lignina & 3.13 & 1.90 & 2.84 & 2.85 \\
CHOt & 78.52 & 76.67 & 79.54 & 77.12 \\
CNE & 23.01 & 28.27 & 22.82 & 12.03 \\
EB (Kcal/Kg MS) & 4539.12 & 4392.53 & 4426.36 & 4504.98 \\
\hline
\end{tabular}

${ }^{1}$ Balances minerales: de acuerdo con las recomendaciones de Valadares et al (9), se determinó la cantidad de sal mineralizada ofrecida. Composición por kilogramo: $160 \mathrm{~g}$ calcio, $60 \mathrm{~g}$ fósforo, $110 \mathrm{~g}$ sodio, 10 $\mathrm{g}$ magnesio, $50 \mathrm{~g}$ azufre, $82 \mathrm{mg}$ cobalto, $800 \mathrm{mg}$ cobre, $120 \mathrm{mg}$ yodo, $3600 \mathrm{mg}$ manganeso, $27 \mathrm{mg}$ selenio, $5200 \mathrm{mg}$ zinc, $4700 \mathrm{mg}$ hierro, 600 mg (máx) de flúor; ${ }^{2} \mathrm{MS}=$ materia seca, $\mathrm{PB}=$ proteína bruta, $\mathrm{EE}=$ extracto etéreo, $\mathrm{MI}=$ material inorgánico, $\mathrm{FDNp}=$ fibra detergente neutra corregida para proteína, $\mathrm{FDA}=$ fibra detergente ácida, $\mathrm{CHOt}=$ carbohidratos totales, $\mathrm{CNE}=$ carbohidratos no estructurales, $\mathrm{EB}=$ energía bruta.

Aproximadamente cada $79 \mathrm{~kg}$ de peso, en promedio, y por un período de cuatro semanas, los animales fueron sometidos a un nivel de alimentación de mantenimiento, fijado en 1.15 veces la exigencia de energía neta sugerida por el NRC (10), correspondiente a $77 \mathrm{Kcal} / \mathrm{Kg} \mathrm{PCV}{ }^{0.75}$ (peso corporal vacío metabólico). Posteriormente, ellos fueron ayunados por un período de 72 horas, después del cual el alimento se aumentó gradualmente hasta alcanzar niveles de alimentación ad libitum. Este manejo fue realizado para obtener las variables asociadas con el metabolismo energético.

Manejo. Antes del inicio del experimento, todos los animales fueron tratados para ecto y endoparásitos y recibieron una dosis inyectable de vitaminas $A, D$ y $E$. Todos los animales fueron pesados al inicio del experimento y con intervalo promedio de 14 días hasta el final del mismo, obteniendo información desde los 11 hasta los 28 meses de edad, aproximadamente. Los pesajes fueron realizados por dos días consecutivos para la determinación del peso vivo medio, siempre en el mismo horario y con ayuno previo de alimento de 12 horas.
Análisis estadístico. Las funciones de crecimiento evaluadas se presentan en la Tabla 2. El ajuste de los datos a cada modelo y las estimativas de los parámetros se realizaron a través del proceso iterativo del algoritmo Marquardt del procedimiento para modelos no lineales PROC NLIN de SAS (11).

Tabla 2. Descripción matemática de los modelos no lineales evaluados.

\begin{tabular}{lc}
\hline \multicolumn{1}{c}{ Modelo } & \multicolumn{1}{c}{ Ecuación $^{\mathbf{1}}$} \\
\hline Logístico & $Y=A\left(1+b \exp ^{-k^{*} t}\right)^{-1}$ \\
Brody & $Y=A\left(1-b \exp ^{-k^{*} t}\right)$ \\
Gompertz & $Y=A \exp \left(-b \exp ^{-k^{*} t}\right)$ \\
von Bertalanffy & $Y=A\left(1-b \exp ^{-k^{*} t}\right)^{3}$ \\
\hline
\end{tabular}

${ }^{1} \mathrm{~A}=$ peso asintótico; $\mathrm{K}=$ índice de madurez; $\mathrm{b}=$ parámetro de integración; $M=$ parámetro de inflexión. Se considera $M={ }^{-1}$ (función logística), ${ }^{1}$ (Brody), 3(von Bertalanffy) y $\infty$ (Gompertz).

Los parámetros de las curvas de crecimiento fueron estimados a partir del peso promedio de los cinco animales obtenido en cada medición, calculado a partir del Procedimiento Summary (PROC SUMMARY) de SAS (11), como sugerido por Mendes et al (7). Finalmente se obtuvieron 40 datos de peso-edad, algunos de los cuales se presentan en la tabla 3 .

Tabla 3. Promedio de peso $(\mathrm{Kg})$, con la respectiva desviación estándar (DE), de machos de la raza Nellore a diferentes edades.

\begin{tabular}{ccccc}
\hline Edad & Peso & DE & Mínimo & Máximo \\
11.6 & 188.5 & 17.64 & 168 & 211 \\
14.1 & 236.5 & 19.83 & 213 & 261 \\
16.4 & 300.8 & 14.06 & 287 & 320 \\
18.2 & 322.4 & 16.79 & 300 & 343 \\
20.1 & 354.8 & 21.32 & 318 & 371 \\
22.0 & 405.2 & 20.63 & 377 & 428 \\
24.9 & 434.4 & 22.56 & 405 & 465 \\
27.4 & 472.0 & 25.58 & 440 & 498 \\
\hline
\end{tabular}

Los criterios considerados para la evaluación de los modelos fueron el cuadrado medio del residuo (o del error) (CME), el coeficiente de determinación $\left(R^{2}\right)$, la dócima de DurbinWatson (DW) y el análisis de los residuos (términos de error). Los residuos fueron graficados frente a la edad, buscando determinar la magnitud en que los modelos sobreestimaron 0 subestimaron ciertas secciones de la curva de peso corporal a través del tiempo, verificando con ello la 
constancia de la varianza del error. Los residuos de cada modelo fueron calculados como la diferencia entre los valores observados y los predichos $\left(e_{i}=y_{i}-\hat{y}_{i}\right)$.

Una vez que el plano nutricional impacta las curvas de crecimiento observadas (Figura 1) y por tanto, la capacidad predictiva de los modelos de crecimiento (curvas de crecimiento estimadas), fue realizada la prueba de Shapiro-Wilk (SW) para verificar el cumplimiento del supuesto de normalidad del conjunto de residuos derivados de cada modelo. La hipótesis nula $(\mathrm{Ho}), \mathrm{Ho}=$ muestra de residuos $\mathrm{X}_{1}, \ldots$ $X_{n}$ proviene de una población normalmente distribuida, fue rechazada cuando el $p$-value fue menor al 5\%, otorgando la menor habilidad predictiva al modelo que originó los respectivos residuos.
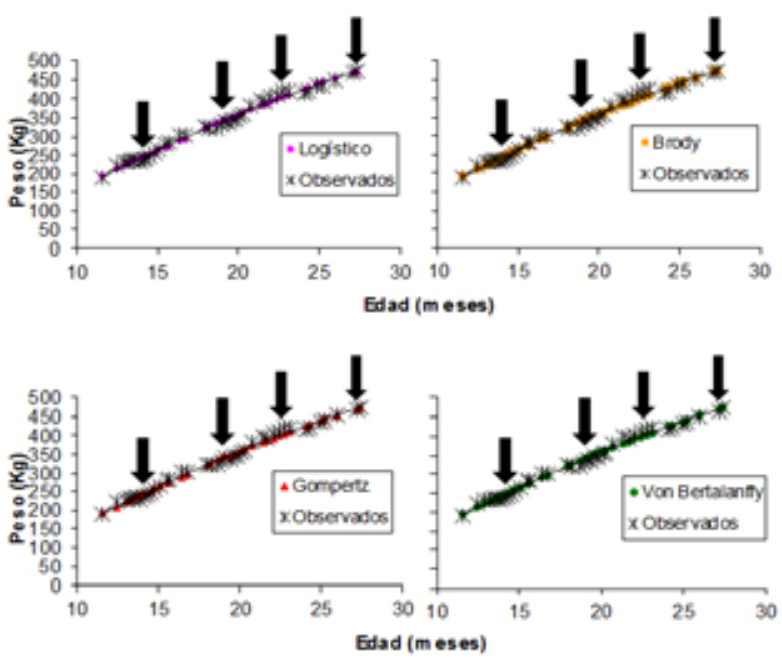

Figura 1. Peso corporal observado y su ajuste por los modelos Logístico, Brody, Gompertz y von Bertalanffy (las flechas indican los momentos en que fue realizada la restricción alimenticia).

\section{RESULTADOS}

Las estimativas de los parámetros, resultantes del ajuste individual de los modelos de crecimiento no lineales a los datos de peso-edad de los animales, con sus respectivos errores estándar, son presentadas en la tabla 4. Dentro de los modelos estudiados, la función de Brody presentó el mayor valor estimado para el parámetro $A$, el cual fue, sin embargo, acompañado de un elevado valor del
Tabla 4. Estimativas para el peso asintótico (A) en $\mathrm{kg}$, constante de integración (b) y tasa de madurez $(k)$ en días ${ }^{-1}$ para diferentes funciones de crecimiento.

\begin{tabular}{lccc}
\hline \multicolumn{1}{c}{ Función } & A (EE $\left.{ }^{1}\right)$ & b (EE) & K (EE) \\
\hline \multirow{2}{*}{ Logística } & 563.1 & 9.6296 & 0.0046 \\
& $(21.03)$ & $(0.7437)$ & $(0.00029)$ \\
Brody & 923.5 & 1.1359 & 0.0010 \\
& $(162.30)$ & $(0.0669)$ & $(0.00028)$ \\
Gompertz & 640.5 & 3.2500 & 0.0028 \\
& $(39.45)$ & $(0.2129)$ & $(0.00029)$ \\
von Bertalanffy & 689.8 & 0.7601 & 0.0022 \\
& $(54.32)$ & $(0.0477)$ & $(0.00029)$ \\
\hline
\end{tabular}

${ }^{1}$ Error estándar

error estándar. Los restantes modelos presentaron un error estándar menor. El modelo de Brody presentó la menor estimativa del parámetro $k$, valores intermedios fueron encontrados en los modelos de Gompertz y von Bertalanffy, en cuanto la función logística resultó en la mayor estimativa. Los errores estándar de todos los modelos estuvieron muy próximos.

El valor de los diferentes criterios de bondad de ajuste, representados por el CME, el $\mathrm{R}^{2}$ y la dócima de DW, para cada uno de los modelos de crecimiento se muestra en la tabla 5. En esta misma tabla se presenta el valor de la prueba de normalidad de SW, y su $p$-value asociado, para los residuos obtenidos de cada modelo de crecimiento. El modelo logístico fue el único que presentó ausencia de normalidad en el conjunto de residuos $(p<0.05)$.

Tabla 5. Evaluadores de calidad de ajuste para diferentes funciones de crecimiento.

\begin{tabular}{ccccc}
\hline Función & CME & $\mathbf{R}^{2}$ & DW & SW* \\
\hline Logística & 71.24 & 99.91 & 0.71 & $\begin{array}{c}0.9430 \\
(0.0438)\end{array}$ \\
Brody & 77.53 & 99.90 & 0.63 & $\begin{array}{c}0.9456 \\
(0.0536)\end{array}$ \\
Gompertz & 73.42 & 99.90 & 0.68 & $\begin{array}{c}0.9462 \\
(0.0562) \\
\text { von Bertalanffy }\end{array}$ \\
& 74.55 & 99.90 & 0.66 & $\begin{array}{c}0.9457 \\
(0.0542)\end{array}$ \\
\hline
\end{tabular}

*Valores entre paréntesis corresponden al p-value asociado a la prueba de normalidad de Shapiro-Wilk.

Las figuras 1 y 2 presentan los valores observados y predichos por todos los modelos y su distribución de residuos, respectivamente. De forma visual, todas las funciones presentaron comportamiento 


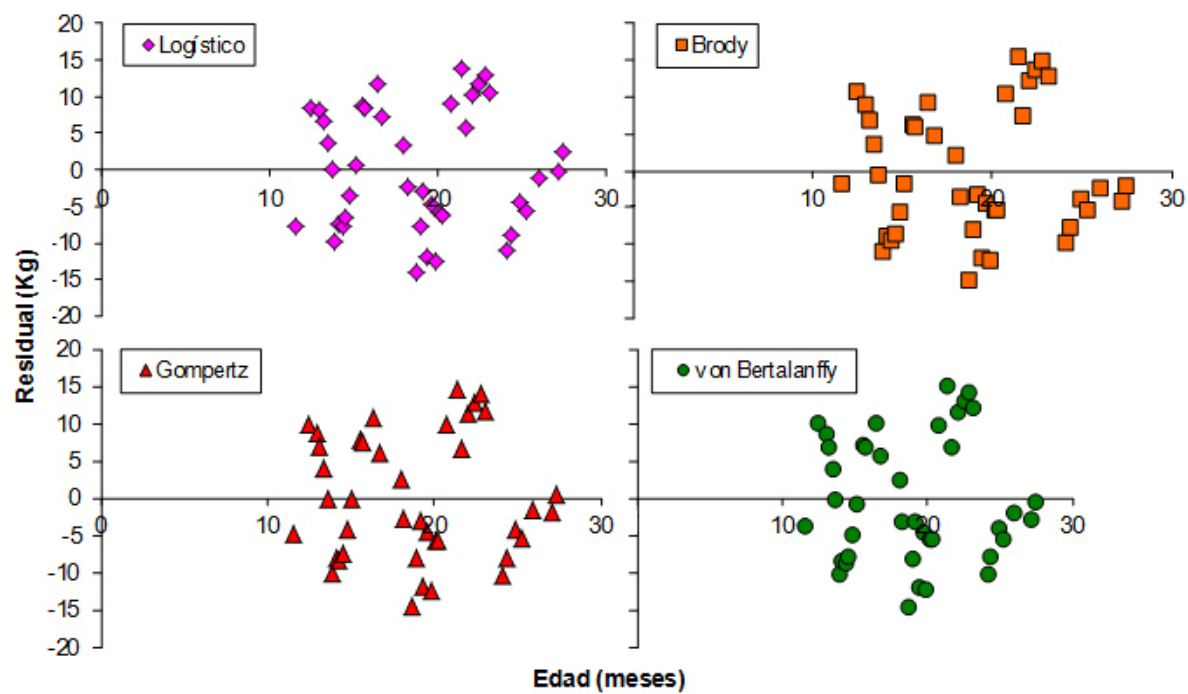

Figura 2. Distribución de los residuos en función de la edad de machos Nellore enteros para diferentes modelos de crecimiento.

similar en su capacidad de ajuste en todo el intervalo de edad contemplado en el trabajo y aparentemente ninguna se destacó por presentar mayor alternancia de los residuos. Se observa que el peso de los animales fue tanto sobreestimado como subestimado en todo el intervalo de edad evaluado.

\section{DISCUSIÓN}

Las ecuaciones de crecimiento utilizadas parten de diferentes supuestos para realizar el ajuste a los datos, como se evidencia por la diferencia en las estimativas realizadas (Tabla 4). Aún así, los valores predichos estuvieron próximos de los reales, lo cual fue confirmado por los diferentes criterios utilizados para evaluar su capacidad de ajuste.

Debido a que la suma de cuadrados total (SCT) es siempre la misma para un conjunto de datos, el valor de la suma cuadrados del error (SCE) depende exclusivamente del ajuste del modelo. Modelos que representen mejor un conjunto de datos ofrecen menores SCE, y por tanto, menores CME. De acuerdo con este criterio, todos los modelos del presente trabajo pueden ser considerados válidos para describir la curva de crecimiento de los machos Nellore en el intervalo de edad contemplado. La misma conclusión puede darse al considerar la proporción de la varianza del peso corporal explicada por el modelo (suma cuadrados del modelo) en relación con la varianza total (SCT), ya que todos las funciones presentaron valores de $\mathrm{R}^{2}$ correspondientes a $99.9 \%$ (Tabla 5).

En relación con la distribución residual, Bergamasco et al (12) indicaron que modelos que presenten distribución alternada en cortos intervalos de tiempo son más deseables que aquellos que la exhiban en intervalos más largos. Esto es, la independencia de los términos de error asigna la mayor capacidad de ajuste a los modelos que registran la menor correlación entre residuos sobre el tiempo. En el presente trabajo se demostró la existencia de autocorrelación serial de primer orden entre residuos, lo cual permite concluir que los modelos aproximaron los datos con errores sistemáticos (Figura 2). La autocorrelación detectada fue positiva, indicando que la diferencia entre residuos fue muy pequeña y que el valor de DW se aproximó a cero (Tabla 5).

La curva de crecimiento observada, resultante de la aplicación de diferentes planos de alimentación, fue la misma para los cuatro modelos evaluados (Figura 1), de forma tal que el cumplimiento del supuesto de normalidad de los residuos dependió del mejor ajuste del modelo a los datos (curva de crecimiento estimada). El modelo 
logístico fue el único que presentó ausencia de normalidad en el conjunto de residuos $(p<0.05)$ bajo las actuales circunstancias experimentales, lo que confirma su mayor tendencia a sobreestimar o subestimar determinados puntos de la curva, en relación con los restantes modelos. La condición de normalidad en los residuos de los modelos de Brody, Gompertz y von Bertalanffy $(p>0.05)$ permite asegurar que la inferencia sobre los parámetros fue adecuada, con independencia de los cambios de peso resultantes de la aplicación de diversos planos de alimentación.

Toda vez que el análisis visual (Figura 1 y 2) y los criterios empleados para evaluar la capacidad de ajuste de los modelos presentaron valores próximos y no destacaron la superioridad de un modelo frente a los demás, excepto por el análisis de normalidad de residuos, la escogencia de la función que mejor describió los datos se basó en la coherencia biológica de los parámetros estimados, tomando como referencia valores reportados en la literatura.

En este trabajo, $\mathrm{A}, \mathrm{b}$ y $\mathrm{K}$ oscilaron entre 563.1 y $923.5,0.7601$ y $9.6296,0.0010$ y 0.0046 , respectivamente (Tabla 4 ). Se confirmó la correlación negativa entre el peso maduro $(A)$ y la tasa de madurez $(K)$. De acuerdo con Silva et al (13), la correlación genética entre los valores de $\mathrm{A}$ y $\mathrm{K}$ indica un antagonismo entre las estimativas de esos parámetros, por tanto, al seleccionar animales buscando mayor tasa de madurez (más precoces), se obtienen animales de menor peso adulto. Valores por debajo a los encontrados en este estudio para A y superiores para $\mathrm{K}$ han sido reportados en ganado Nellore, machos y hembras, por Mendes et al (7), Silva et al (1,6, 13), Santoro et al (2) y Brasão y Silva (14). Las estimativas de peso adulto encontradas en estos trabajos fluctuaron entre 240.01 y $487.58 \mathrm{~kg}$, en cuanto la tasa de madurez varió entre 0.0019 y 0.0092 .

Las mayores estimativas para A y las menores estimativas para $\mathrm{K}$ obtenidas en este trabajo, en relación con los reportes de la literatura, está indicando que los animales alcanzarán peso asintótico en edad más avanzada en razón de su desarrollo más lento. Herrera et al (4) indican que grupos animales con mayor valor en el parámetro $A$ son menos precoces al registrar menor valor en el parámetro $\mathrm{K}$, presentando con ello una menor velocidad de crecimiento para llegar al peso asintótico a partir del peso inicial. Si bien el peso maduro es independiente de fluctuaciones de tamaño en el corto plazo causadas por factores climáticos $\mathrm{y} / \mathrm{o}$ aporte nutricional (15), su predicción a través de curvas de crecimiento si puede ser afectada por variaciones ambientales temporales.

Los animales del presente estudio experimentaron períodos de alimentación restricta en cuatro fases experimentales diferentes, con duración de cuatro semanas cada uno, y posterior sometimiento a períodos de ayuno de 72 horas, donde registraron pérdidas de peso significativas (entre 5.5 y $15.7 \mathrm{~kg}$, correspondientes a 1.7 y $4.89 \%$ del peso), lo que explica porque los pesos maduros y las tasas de madurez presentaron divergencia con respecto a los reportes de la literatura.

Se concluye que el modelo de Brody fue el que mejor ajustó los datos del presente estudio porque, además de presentar buen ajuste matemático, registró una mayor coherencia biológica en el peso maduro estimado, tomando en consideración los pesos adultos reportados por Vaca (16). Según este autor, las vacas adultas de la raza Nellore pueden llegar a pesar 800 kg, mientras los toros sobrepasan con facilidad los $1000 \mathrm{~kg}$. Forni et al (17), considerando que el peso a la madurez es posterior al peso de sacrificio, señalaron la validez de los modelos de crecimiento como alternativa para su predicción y su valioso aporte en los programas de selección genética.

En relación con los modelos disponibles para caracterizar datos de crecimiento, el propuesto por Brody ha sido el más frecuentemente aplicado en ganado vacuno $(4,18)$. Su mejor comportamiento, interpretación biológica de los parámetros y menor error medio de predicción, en 
relación con las funciones von Bertalanffy, Gompertz y Logística, fue confirmada en los trabajos de Silva et al (6), Mendes et al (7), Santoro et al (2) y Brasão y Silva (14) con ganado Nellore. Silva et al (6) encontraron que los modelos de von Bertalanffy, Gompertz y Logístico subestimaron el peso adulto de los animales, posiblemente por la poca habilidad de esas funciones para ajustar el peso inicial, comprometiendo el proceso de estimación de la tasa de madurez y consecuentemente del peso adulto.

Agudelo et al (5) y Freitas (3) informaron sobre estudios comparativos en los que estos tres modelos matemáticos sobrestimaron los pesos a edades tempranas, en tanto que reportes citados por Ramírez et al (18) observaron que el modelo de Brody sólo realizó una ligera sobrevaloración en edad temprana y una adecuada predicción del peso adulto. Contrariamente, Silva et al (1) concluyeron que la función de Brody superestimó el peso asintótico y que la función de Gompertz fue la que registró mayor porcentaje de convergencia. También Bergamasco et al (12) concluyeron que el modelo Brody presentó una estimativa de peso asintótico superior al convencional en la descripción del crecimiento de hembras de raza Holstein. Silva et al (6) indicaron que la función de Brody es la más deseable cuando un estudio de crecimiento no incluye los primeros años de desarrollo o solamente dispone de un pequeño número de pesajes en este período, situación registrada en el presente trabajo.

El crecimiento animal sigue una curva sigmoidea a través de la cual la tasa de crecimiento varía con la edad y llega a un punto en el que declina a cero; en este punto se alcanza la asíntota y el animal logra el peso maduro. Agudelo et al (5) señalan que el animal crece en forma lenta durante el primer mes posparto, después inicia una fase de rápido crecimiento hasta alcanzar la pubertad y posteriormente disminuye su velocidad de crecimiento hasta estabilizar el peso adulto. En concepto de los autores, esta dinámica es la responsable de que entre los 6 a 9 meses se presente un primer punto de inflexión, que se corresponde con el valor máximo de la curva de crecimiento corriente. En el presente trabajo no fue posible visualizar este primer cambio de curvatura, ya que los datos de peso fueron obtenidos desde el primer año, aproximadamente (Figura 1).

El peso a la madurez, que representa el peso corporal genético, es alcanzado cuando cualquier incremento en peso vivo, que no sea llenado gastrointestinal, sólo corresponde a incremento lipídico (15). Taylor (19) definió peso maduro como aquel en el cual el animal alcanza $20 \%$ de grasa corporal. Considerando diferentes razas, se acepta que el peso a la madurez se alcanza a los cinco años (15).

Silva et al (6) señalaron que la dificultad de ajuste (bajos porcentajes de convergencia) de los modelos no lineales se debe a la utilización de animales jóvenes para la estimación del peso adulto y también a la falta de datos en períodos críticos de la curva, que están alrededor del punto de inflexión. De acuerdo con Santoro et al (2), el punto de inflexión estaría poco definido cuando la edad máxima de colecta de datos es de dos años, porque aún falta que los animales continúen desarrollándose. Los autores entonces justifican el acompañamiento del peso hasta edades superiores en animales cebuínos para no afectar el cálculo del punto de inflexión real. Según información referenciada por Arango y Vleck (15), la estimación del peso maduro a través de curvas de crecimiento sólo es confiable cuando los datos de peso existentes corresponden a una edad superior a los 4.5 años. Los trabajos citados en la literatura $(1,2,6,7,14)$ sólo ajustaron los datos de crecimiento desde el nacimiento hasta una edad máxima de 24 meses, en tanto que el presente estudio sólo se extendió por un período adicional de 3.5 meses, lo cual estaría explicando la ausencia de punto de inflexión en la figura 1.

Por tratarse de animales en régimen de confinamiento y suplementados, se verificó que las medias de peso corporal registradas a diferentes edades fueron superiores a los valores medios observados por Giolo et al (20) y Silva et al (13) en edades 
similares con animales en pastoreo. Esto también permite proponer que el mayor peso asintótico predicho por los modelos en el presente estudio es resultado de mayores planos de alimentación, donde a edades equivalentes, la media de peso ajustada presenta un valor mayor. Lo anterior se verifica al comparar los presentes resultados con los obtenidos por Silva et al (1). La media de peso ajustada por los diferentes modelos en ese estudio, con datos derivados exclusivamente de animales en pastoreo, fue menor a 320 $\mathrm{kg}$ a los dos años de edad, mientras que en este trabajo, a la misma edad, el valor predicho superó los $425 \mathrm{~kg}$. La evolución del aumento de peso vivo a lo largo de la vida de un animal es por tanto un fenómeno complejo que depende del genotipo del animal, de factores ambientales como el manejo, el estado de salud, el clima y principalmente, la alimentación (5).

En conclusión, la verificación de la convergencia de los modelos no garantiza que los parámetros estimados se mantengan en los límites biológicamente aceptables.
Esto es, de acuerdo con el CME, el $\mathrm{R}^{2}$ y la dócima de DW, todos los modelos evaluados en este trabajo ajustaron apropiadamente los datos peso-edad; no obstante, por la interpretación biológica de los parámetros, el modelo Brody fue el que mejor describió el crecimiento de machos Nellore criados en confinamiento, con suplementación alimenticia y bajo el manejo restrictivo desde el punto de vista nutricional al que fueron sometidos. Sin la ocurrencia de estas restricciones, y considerando que los animales fueron alimentados con una dieta que presentó una relación forrajeconcentrado fluctuando entre 60:40 y 80:20, seguramente los parámetros estimados estuvieran más próximos o inclusive superarían en precocidad y eficiencia aquellos reportados con esta raza en régimen de pastoreo.

\section{Agradecimientos}

A la Fundación Universitaria San Martín, por el apoyo financiero para la ejecución de este trabajo.

\section{REFERENCIAS}

1. Silva NAM, Aquino $L H$, Silva $F F$, Oliveira AIG. Curvas de crescimento e influência de fatores não-genéticos sobre as taxas de crescimento de bovinos da raça nelore. Ciênc Agrotec 2004; 28: 647-654

2. Santoro KR, Barbosa SBP, Brasil LHA, Santos ES. Estimativas de parâmetros de curvas de crescimento de bovinos zebu, criados no estado de Pernambuco. R Brás Zootec 2005; 34: 2262-2279.

3. Freitas AR. Estimativas de curvas de crescimento na produção animal. EMBRAPA [en línea] 2007 [fecha de acceso 20 de agosto de 2010]; Documentos 68. URL disponible en: http://www.cppse.embrapa.br/08 0servicos/070publicacaogratuita/ documentos/Documentos68.pdf/view.
4. Herrera AC, Vergara OD, Cerón-Muñoz MF, Agudelo DA, Arboleda EM. Curvas de crecimiento en bovinos cruzados utilizando el modelo Brody. Livestock Research for Rural Development [en línea] 2008 [fecha de acceso 10 de septiembre de 2010]; 20 (140). URL disponible en: http://www.Irrd.org/ Irrd20/9/herr20140.htm.

5. Agudelo DA, Cerón-Muñoz MF, Restrepo LF. Modelación de las funciones de crecimiento aplicadas a la producción animal. Rev Col Cienc Pec 2008; 21:39-58.

6. Silva FF, Aquino $\mathrm{LH}$, Oliveira AI. Influência de fatores genéticos e ambientais sobre as estimativas dos parâmetros das funções de crescimento em gado nelore. Ciênc Agrotec 2001; 25: 1195-1205. 
7. Mendes PR, Aquino LH, Muniz JÁ. Estudo da curva de crescimento de fêmeas da raça nelore. Ciênc Agrotec 2001; 25: 958-968.

8. Inzunza JC. Clasificación de los climas de Köppen. Ciencia Ahora 2005; 15:1-14.

9. Valadares Filho SC, Rodrigues $P$, Magalhães K. Exigências nutricionais de zebuínos e tabelas de composição de alimentos. 1 ed. Viçosa: Universidad Federal de Viçosa, 2006.

10. National Research Council - NRC. Nutrient requirements of beef cattle. 7 ed. Washington, D.C: National Academy Press, 2000.

11. SAS/STAT: Guide for Personal Computer [programa de ordenador]. Versión 8.2. Cary (NC): SAS Institute Incorporation; 2001.

12. Bergamasco AF, Aquino LH, Muniz JA. Estudo da curva de crescimento de fêmeas da raça Holandesa. Ciênc Agrotec 2001; 25: 235-242.

13. Silva FF, Aquino LH, Oliveira AI. Estimativas de parâmetros genéticos de curva de crescimento de gado nelore (Bos indicus). Ciênc Agrotec 2002; (Edição Especial):1562-1567.

14. Brasão LC, Silva HD. Ajuste de modelos não lineares à curva de crescimento média de fêmeas da raça Nelore [en línea] sf. [fecha de acceso 28 de julio de 2010]. URL disponible en: http://www.posgraduacao.ufla.br/ gauss/congresso/11seagro/conteudo/ arquivos/83.pdf.
15. Arango JA, Van Vleck LD. Size of beef cows: early ideas, new developments. Genet Mol Res [en línea] 2002; 1(1):51-63. [fecha de acceso $14 \mathrm{de}$ octubre de 2010]. URL disponible en: http://www.funpecrp.com.br/gmr/ year2002/vol1-1//pdf/gmr0021.pdf.

16. Vaca JL. Análisis de dos sistemas de producción ecológica utilizando novillos nelore y criollo chaqueño en el área integrada de SantacruzBolivia. [Tesis Doctoral]. España: Universidad de Córdoba. [en línea] 2003 [fecha de acceso 28 de julio de 2010]. URL disponible en: http:// helvia.uco.es/xmlui/bitstream/ handle/10396/353/13208421. pdf?sequence $=1$.

17. Forni $S$, Piles $M$, Blasco $A$, Varona $L$, Oliveira HN, Lôbo RB, Albuquerque LG. Genetic análisis of growth curve parameters applying a von Bertalanffy function to beef cattle longitudinal data. 8th World Congress on Genetics Applied to Livestock Production, Belo Horizonte, MG, Brasil. 2006.

18. Ramírez EJ, Cerón-Muñoz MF, Herrera AC, Vergara OD, Arboleda EM, Restrepo LF. Crecimiento de hembras cruzadas en el trópico Colombiano. Rev Col Cienc Pec 2009; 22:642-647.

19. Taylor CS. Use of genetic size-scaling in evaluation of animal growth. J Anim Sci 1985; 61: 118-143.

20. Giolo SR; Henderson $\mathrm{R}$, Demétrio CGB. Mixed-effects growth curves in the evaluation of nellore sires. Sci Agric 2009; 66: 84-89. 